\title{
Percepción de los docentes sobre las buenas prácticas con un aplicativo móvil para la enseñanza de matemáticas ${ }^{*}$
}

\author{
RICARDO JAVIER NAVARRO FERNÁNDEZ \\ MARÍA VANESSA VEGA VELARDE \\ ENRIQUE CHIROQUE LANDAYETA ${ }^{* * * * *}$ \\ CAROL RIVERO PANAQUÉ ${ }^{* * * * * *}$
}

Grupo AVATAR - Pontificia Universidad Católica del Perú

Recibido el 13-09-2017; primera evaluación el 15-12-2017;

segunda evaluación el 02-01-2018; aceptado el 27-02-2018

\section{Resumen}

Este estudio busca identificar las nociones que los profesores de instituciones educativas públicas tienen sobre el concepto de buenas prácticas docentes, especialmente, respecto al uso de la tecnología en el salón de clase. Asimismo, se explora las percepciones sobre la utilidad del uso de un aplicativo móvil para enseñar matemáticas. Se inicia con una revisión teórica que delimita el concepto. A partir de esta, se diseñó una guía de entrevista para conocer la percepción de los profesores.

\footnotetext{
Esta investigación fue realizada dentro del Proyecto Oráculo Matemágico, financiado por Fundación Telefónica.

** Licenciado en Psicología Educacional por la Pontificia Universidad Católica del Perú (PUCP). Posee una diplomatura en monitoreo y evaluación de programas por la PUCP. ricardo. navarro@pucp.pe

${ }^{* * *}$ Bachiller en Psicología Educacional por la Pontificia Universidad Católica del Perú. Asistente de investigación en el Grupo AVATAR PUCP. Contacto: vanessa.vega@pucp.pe

${ }^{* * * *}$ Profesor a tiempo completo del Departamento de Diseńo Gráfico de la Facultad de Arte y Diseño de la Pontificia Universidad Católica del Perú. coordinador general del grupo AVATARPUCP, que se centra en el desarrollo e investigación del uso de nuevas tecnologías en el contexto educativo. Coordinador de la especialidad de Diseño Gráfico de la PUCP. Contacto: echiroque@ pucp.edu.pe

Profesora asociada del Departamento de Educación de la Pontificia Universidad Católica del Perú. Doctoranda en Ciencias de la Educación por la PUCP y máster en Ingeniería de Medios para la Educación por la Unión Europea. Con estudios de Maestría en Investigación Psicológica (PUCP). Licenciada en Psicología y Educación. Contacto: crivero@pucp.pe
} 
sobre las buenas prácticas docentes y el uso de tecnologías. Los resultados apuntan a que los docentes identifican el concepto de buenas prácticas, pero no reconocen casos concretos donde estas se utilicen. Por otro lado, identifican las ventajas de las tecnologías y su uso en el aula, reconociendo aspectos importantes para la enseńanza

Palabras clave: tecnología y educación, tecnología educativa, matemáticas y enseñanza de matemáticas.

\section{Teacher's perception on good practices with a mobile application for teaching mathematics}

\section{AbStract}

This study aims to identify public schools teacher's perceptions of the Good Teaching Practices, especially the use of technology in the classroom. Additionally, the study identify teacher's perception concerning the use of a mobile app to teach mathematics. The study begins with a theoretical review that delimit the concept. From this review, an interview guide was designed to identify teacher's perceptions of good teaching practices and the use of technologies in the classroom. The results suggests that teachers identify Good Teaching Practices in a conceptual approach, but not in a practical approach. In addition, they acknowledge the benefits of technologies for their teaching practices, recognizing the importance for teaching mathematics.

Keywords: Technology and Education, Educative Technology, Mathematics and Teaching of Mathematics.

\section{Percepçáo de professores sobre boas práticas com um aplicativo móvel para ensino de matemática}

\section{Resumo}

Este estudo busca identificar as noçóes que os professores das instituiçôes educacionais públicas têm sobre o conceito de boas práticas de ensino, especialmente com o uso da tecnologia na sala de aula. Também explora as percepçóes sobre a utilidade de usar uma aplicação móvel para ensinar matemática. Começa com uma revisão teórica que delimita o conceito. A partir disso, um guia de entrevista foi projetado para conhecer a percepção dos professores sobre boas práticas de ensino e o uso de tecnologias. Os resultados sugerem que os professores identifiquem o conceito de boas práticas, mas eles não reconhecem casos concretos em que são usados. Por outro lado, eles identificam as vantagens das tecnologias e seu uso na sala de aula, reconhecendo aspectos importantes para o ensino.

Palavras-chave: tecnologia e educação, tecnológica educacional, matemática e ensino de matemática. 


\section{INTRODUCCIÓN}

Todo sistema educativo tiene como finalidad formar individuos que puedan responder adecuadamente a las necesidades de un contexto específico (Lourenco, 2015), sea este laboral, deportivo, artístico, académico, o de otro tipo. Esto se cumple también, al nivel de la educación como política de estado, ya que las sociedades, en general, también tienen requerimientos propios (y complejos) que deben ser abordados por todos sus integrantes. En ese sentido, todo sistema educativo nacional tiene por objetivo central la formación de ciudadanos que puedan integrarse satisfactoriamente en su contexto cultural, desarrollando al máximo su potencial cognitivo y social (Piaget, 1970, 1973; Lourenco, 2015). Sin embargo, los cambios constantes impuestos por los avances tecnológicos y los requisitos de especialización del mercado exigen líneas de formación a tener en cuenta para la educación del futuro, lo que supone replantear algunos supuestos establecidos en los métodos clásicos de enseñanza.

Independientemente de cual sea el contexto objetivo de formación de un proceso educativo particular, uno de los roles más importantes en cualquiera de estos sistemas es el del docente, que es el encargado de orientar el proceso de aprendizaje del estudiante (Murillo, Martínez y Hernández, 2011). Así, si bien son muchos los elementos que deben ser tomados en cuenta para el éxito de cualquier proceso de enseñanza-aprendizaje, el desempeño del docente y la calidad de sus metodologías, estrategias y dominio temático, son elementos que no pueden ser dejados de lado.

De entre los elementos fundamentales del trabajo docente mencionados en el párrafo anterior, uno que ha sido poco estudiado, debido a su complejidad y novedad, es el de buenas prácticas docentes (BPD). Este constructo ha sido utilizado en diversos contextos con acepciones no siempre idénticas. No obstante, de entre las distintas definiciones esbozadas, se pueden rescatar características compartidas.

En un principio, el constructo de Buenas Prácticas se originó en el ámbito empresarial, donde se buscaba rescatar experiencias o modelos que hubieran tenido éxito al ser utilizados en contextos determinados (Abdoulaya, 2003, De Pablos y Gonzales, 2007). Así, el término era utilizado de forma muy general como medio de calificar "cualquier actividad que ofrece buenos resultados en el contexto en el que se utiliza» (De Pablos y Gonzales, 2007, p. 9). En esa misma línea, Escudero (2009) indica que las Buenas Prácticas son «una serie de atributos o características referidas a formas de hacer exitosas en el logro de objetivos» (p. 13). 
En ese sentido, las Buenas Prácticas en el ámbito empresarial son entendidas como experiencias que han tenido buenos resultados en la situación en que fueron utilizadas (Abdoulaye, 2003; De Pablos y Gonzales, 2007; Escudero, 2009). Evidentemente, esta aproximación conceptual es bastante vaga, pero, además, presenta la dificultad de tener una orientación empresarial que, en ocasiones, puede oponerse a las necesidades particulares del contexto educativo. Por esta razón, aquellos autores que buscan introducir el término al ámbito de la educación suelen agregar a esta definición la condición de que este tipo de prácticas deben enfocarse en lograr aprendizajes en los estudiantes, así como su desarrollo integral. Por ejemplo, autores como Arnaiz (2011), Colás y Casanova (2010) y Escudero (2009) proponen que las buenas prácticas deben orientarse a mejorar y compartir experiencias positivas en enseñanza, con la finalidad de que el estudiante aprenda y mejore su rendimiento académico. Arnaiz (2011) refiere que "para avanzar hacia la consecución de buenas prácticas cada centro tendrá que analizar su realidad, planificar su plan de mejora, definir las estrategias que pueden impulsar este cambio y evaluar lo realizado» (p. 32). Colás y Casanova (2010) comparten dicha visión y añaden que «las buenas prácticas implican cambios y mejoras» (p. 125). Por último, Colás y Casanova (2010) proponen el uso de nuevas tecnologías para mejorar las prácticas educativas.

Por otro lado, Vidal y Morales (2009) refieren que las BPD deben ser abordadas como experiencias educativas que facilitan el desarrollo de actividades de aprendizaje, cuyo objetivo sea lograr de manera eficaz objetivos previstos (normalmente en un currículo). Así, también es necesario reconocer que un aspecto crucial de las BPD es que permiten pasar por un proceso de sistematización de experiencias (documentarlas, analizarlas y criticarlas), lo que permite identificar aspectos positivos y negativos (Escudero, 2009).

En síntesis, las BPD pueden conceptualizarse como procedimientos o métodos de acción docente que generan un cambio positivo en una situación académica particular (De Pablos y González, 2007; Hernández, 2001, citado en Escudero, 2009) y que se encuentran orientados a un objetivo de aprendizaje adecuadamente delimitado y estructurado, que es posible replicar en otros contextos, así como evaluar y medir (Villa, 2006, citado en De Pablos y González, 2007).

Para la elaboración y/o identificación de las BPD, diversos autores han postulado características y principios adicionales a los ya señalados. Por ejemplo, varios autores señalan que las BPD deberían proponer actividades que generen interacción entre el docente y los estudiantes, todas estas contextualizadas según las características y necesidades propias del grupo de alumnos 
con el cual se está trabajando (Chickering y Gamson, 1987; Epper y Bates, 2004; Marqués, 2002, citado en Vidal y Morales, 2009).

Por otro lado, De Pablos y Jiménez (2007) hacen énfasis en el carácter innovador de las BPD, argumentando que el desarrollo de una buena práctica responde a una visión de desarrollo constante. En este sentido, las BPD responden a una necesidad de "querer avanzar», que no está limitada únicamente a los métodos clásicos de enseñanza, sino que hace referencia a la transformación de la propia conceptualización de las BPD a través del tiempo, las cuales se adaptan a las necesidades temporales y contextuales de los estudiantes y el docente.

En esa línea, Epper y Bates (2004) y De Pablos y Jiménez (2007) señalan la importancia del componente «innovador» de las BPD, a partir del cual se han realizado distintas propuestas en sus aplicaciones y diseños. En ese sentido, una de las innovaciones más importantes que se vienen realizando en los últimos ańos es la integración de las tecnologías de la información y la comunicación (TIC) en el salón de clase.

$\mathrm{Al}$ respecto, Graells (2013) y Prieto, Quiñones, Ramírez, Fuentes, Labrada, Pérez Hechavarría y Montero (2011), señalan que la presencia y el uso de las TIC es innegable y necesario, luego de que estas se han introducido en distintos aspectos de nuestra sociedad actual. La educación no puede ser una excepción a esto, dado que su función es justamente preparar a los estudiantes para integrarse a un mundo que se encuentra en constante cambio. Esta visión del uso de TIC en el contexto educativo es compartida por Colás y Casanova (2010), quienes proponen este tipo de herramientas como materiales fundamentales para las BPD. Por esta razón, se han realizado grandes esfuerzos para que las TIC sean incluidas en los modelos de enseñanza en colegios y escuelas (Noguera, 2015).

Las TIC facilitan la construcción de nuevo conocimiento, dado que fungen como mediadores del aprendizaje, fomentando un contexto atractivo e interactivo para el estudiante (Mikre, 2011). Debido a estos componentes útiles para una pedagogía constructivista en los últimos años, se han realizado diversas iniciativas para incorporar las TIC en el salón de clase (Mikre, 2011; Voogt, 2003). De estas experiencias se rescata que generan un mayor interés de parte de los estudiantes hacia los temas que se enseñan en el aula, así como mayor participación y protagonismo (Mikre, 2011).

Entre las TIC de mayor alcance en la actualidad, se encuentran los aplicativos para móviles (Fojtik, 2014). Este tipo de tecnología tiene un gran potencial para transformar la educación y la práctica docente (Keengwe y Bhargava, 2014), pero para ello deben ser implementadas de manera relevante 
al contexto social y cultural de los estudiantes (Keengwe y Bhargava, 2014; Fojtik, 2014).

Dado que pueden existir desafíos para el diseño e implementación de este tipo de tecnologías en países que no están acostumbrados a esta clase de innovaciones, se hace relevante el diseño de proyectos educativos que las incorporen. Lamentablemente, muchos de estos tipos de proyectos no han tenido los resultados esperados. Esto puede deberse a diversos factores, pero una de las causas más recurrentes suele ser que el docente no es instruido respecto de cómo deben utilizarse estas tecnologías. Si bien estas son herramientas útiles que pueden servir en la enseñanza, no tienen un impacto adecuado si los actores educativos que deben utilizarlas no comprenden adecuadamente cómo hacerlo.

Por ello, Fundación Telefónica y la Pontificia Universidad Católica del Perú, ante la necesidad de la implementación de las TIC en la educación, han desarrollado el aplicativo móvil Oráculo Matemágico, una herramienta tecnológica diseñada para facilitar la enseńanza de las matemáticas. Este aplicativo presenta al niño problemas matemáticos especialmente diseñados en función a investigación psicopedagógica; asimismo, lo hace de modo atractivo, ya que integra el entrenamiento de habilidades matemáticas con un videojuego de cartas intercambiables que estimula su práctica.

Oráculo Matemágico está diseñado para que el estudiante practique y resuelva problemas de aritmética, geometría, estadística y álgebra, gamificando la experiencia de resolución de los mismos. Asimismo, los problemas diseńados para este fin presentan una estructura que promueve el razonamiento lógico por parte de los estudiantes, evitando una redacción que favorezca la utilización mecánica de algoritmos descontextualizados.

Finalmente, el diseño del aplicativo móvil «Oráculo Matemágico» consideró, también, los procesos motivacionales involucrados en la dinámica de enseñanza-aprendizaje de las matemáticas en las aulas de clase. De ese modo, conscientes del poco interés o ansiedad que la enseńanza tradicional de esta materia ha generado en muchos estudiantes, se buscaron medios para motivar y mantener el interés en los estudiantes, por lo que el diseño del aplicativo consideró las teorías de motivación más relevantes en la actualidad.

En función de todo lo anterior, el objetivo del presente estudio es conocer qué entienden los docentes de Educación Básica Regular de nivel primaria por BPD, en el salón de clase, así como, sus percepciones sobre el uso de un aplicativo móvil (Oráculo Matemágico) en las clases de matemática. 


\section{Metodología}

El presente estudio tiene un diseño de investigación cualitativa, de recojo de información a partir de entrevistas. Asimismo, este estudio es de tipo descriptivo, pues se busca detallar cómo los docentes perciben tanto las buenas prácticas docentes como las TIC, así como su percepción sobre el aplicativo móvil Oráculo Matemágico.

\subsection{Participantes}

Se seleccionó a seis docentes (cinco mujeres y un hombre) de Educación Básica Regular del nivel de Primaria, quienes, al momento de la investigación, enseñaban el curso de matemáticas en los grados de $50^{\circ}$ y $6 .^{\circ}$ de primaria. La edad de los participantes varía entre los 45 y 55 ańos de edad, con una experiencia laboral entre 15 y 32 ańos. Los docentes participantes habían llevado un curso virtual sobre el uso del aplicativo Oráculo Matemágico en el aula y se tuvo en cuenta que los entrevistados hubieran obtenido notas altas en el mismo.

Todos los docentes pertenecen a instituciones educativas públicas de las Unidades de Gestión Educativa Local (UGEL) 1 y 5. Además, todos habían utilizado el aplicativo Oráculo Matemágico en sus clases.

\subsection{Recolección de información}

Se realizó la recolección de la información por medio de entrevistas. El objetivo de las entrevistas fue conocer qué entienden los docentes sobre BPD en el salón de clase y sus percepciones sobre la funcionalidad de un aplicativo móvil (Oráculo Matemágico) en las clases de matemática.

Se diseñó una guía de entrevista semiestructurada que estaba dividida en tres áreas:

- Buenas prácticas docentes: En esta área, se buscó recoger la información sobre la definición de buenas prácticas por parte de los profesores, qué consideran que es una buena práctica, si es importante tener buenas prácticas en el salón de clase y sobre qué estrategias utilizaban en el aula.

- Buenas prácticas docentes con tecnología: En esta área, se indagó sobre las buenas prácticas con el uso de tecnologías. Para esto, se preguntó sobre los materiales que utilizaban en sus clases, si era necesario utilizar tecnologías en el aula de clase y cómo incorporaban el uso de tecnologías en el aula.

- Percepciones sobre el aplicativo móvil (Oráculo Matemágico): Esta área buscaba recoger las opiniones de los docentes sobre un aplicativo móvil 
para la enseñanza de matemáticas (Oráculo Matemágico), y conocer su experiencia con el aplicativo (cómo lo integraban a sus clases, en qué momento, cómo recibieron sus estudiantes el aplicativo en clases, beneficios y retos sobre el uso del aplicativo móvil, y qué estrategias utilizaron para poder usarlo en clases de matemática).

\subsection{Procedimiento}

Se seleccionó seis docentes — tres por cada UGEL— que obtuvieron puntajes altos en un curso virtual sobre el uso del aplicativo Oráculo Matemágico. Se realizó el contacto con las instituciones educativas para obtener la autorización de las autoridades y los docentes. Luego, se procedió a coordinar con los docentes el día y hora de la entrevista.

Todas las entrevistas fueron realizadas en cada Institución Educativa a la que pertenecen los docentes y tuvieron una duración de una hora, aproximadamente. Además, todas las entrevistas fueron grabadas y luego transcritas. La información recogida fue analizada por los investigadores, quienes discutieron las categorías establecidas y se llegó a un consenso sobre la codificación de la información recogida. La codificación fue realizada con el programa Atlas-ti.

\section{Resultados Y Discusión}

Con relación a la definición que los profesores tienen sobre buenas prácticas docentes, se puede señalar que relacionan este concepto con habilidades prácticas, como la higiene, la salud, entre otros. Asimismo, contemplan las BPD como estrategias orientadas a mejorar los aprendizajes de los estudiantes:

Entrevistador (E): «¿Qué entiende por buenas prácticas docentes?»

Participante (P): «Bueno entiendo que es la manera de ejecutar o desarrollar un tema, ¿no? para darle de repente más facilidad a los alumnos de que puedan entender un determinado tema a trabajar o a desarrollar en clase» (participante $3)$.

$\mathrm{E}$ : ¿¿Qué entiende por buenas prácticas docentes?»

$\mathrm{P}$ : «Bueno es un término que generalmente está haciéndose nuevo para identificar aquellas actividades en la que nos resulta más exitoso llegar a que los chicos logren aprendizajes duraderos» (participante 1).

E: «¿Qué entiende por buenas prácticas docentes?»

P: «Sí, se ha escuchado y se sigue escuchando ahora porque el profesor tiene que avanzar un poco más, ¿no? o sea, no lo clásico que siempre hacíamos 
donde el niño aprende no sé por qué y ahí me quedo, tengo que buscar otra manera, innovar, investigar, buscar que alguien me ayude o yo mismo crear para que sea una buena práctica» (participante 2).

E: «¿Qué entiende por buenas prácticas docentes?»

P: «Ah, por buenas prácticas yo entiendo de que el profesor tiene que aplicar estrategias para poder llegar al niño, preparándose» (participante 4).

Esta visión de BPD es similar a la propuesta por De Pablos y Jimenez (2007) y Escudero (2009), en la que se identifica una experiencia exitosa que sirve como estrategia para mejorar el desempeńo y aprendizajes de los estudiantes. Asimismo, se relaciona con conceptos más conocidos en la pedagogía, como son las estrategias de enseñanza. Esto podría implicar que los docentes identifican cierta relación entre las BPD y las estrategias a nivel conceptual, lo que podría darle mayor validez educativa a las $\mathrm{BPD}$, pues se distancian de la esencia empresarial con la que este constructo se originó y se les otorga un marco conceptual más pedagógico. Sin embargo, si bien las estrategias pedagógicas y las BPD pueden tener ciertas similitudes en la percepción de los docentes, las estrategias pueden ir más allá, pues son procedimientos que siguen teniendo en cuenta el contexto y las necesidades del estudiante, para alcanzar un objetivo de aprendizaje determinado (Monereo, 2000). Esta perspectiva podría ser agregada a las BPD, generando mejoras en la práctica docente.

Por otro lado, si bien los docentes concuerdan con diversos autores sobre lo que es una BPD a nivel conceptual, los ejemplos que brindan respecto de estos apuntan más a actividades que no necesariamente se relacionan con el quehacer docente, sino con quehaceres fuera del aula o con estrategias a desarrollar para concursos promovidos por el Ministerio de Educación (Minedu):

E: «QQué entiende sobre buenas prácticas docentes?»

$\mathrm{P}:$ «Es cuando nosotros hacemos nuestras clases y lo mejor que hemos desarrollado lo presentamos ya sea para un concurso en el Minedu, a veces ponen en la plataforma que van a hacer un concurso de buenas prácticas docentes, entonces ahí lo llevamos y lo desarrollamos» (participante 5).

E: «¿Qué otra sería una buena práctica?»

$\mathrm{P}:$ «Acá también en la institución, trabajar con biohuertos, lo que es la naturaleza, evitar la contaminación del ambiente» (participante 6).

Sin embargo, también se menciona la utilidad que pueden encontrarle para el aprendizaje de sus estudiantes, donde se señala la importancia de estos materiales para fomentar el error y aprender a partir de ellos:

«Lo que me gusta del programa en sí, porque también es una buena práctica docente en la aplicación del Oráculo Matemágico, es que el niño aprende de su error, de lo que se equivoca (participante 2). 
A partir de esto, se puede inferir que la mayoría de los docentes no concibe las actividades que realizan en el aula de clase a partir del marco conceptual de BPD que conocen, sino que estas se consideran simplemente como estrategias o actividades interactivas para el estudiante, lo que podría reforzar la idea que los docentes conciben a las BPD dentro del marco de estrategias de enseñanza.

De ese modo, los docentes pueden poner en práctica actividades que podrían ser consideradas $\mathrm{BPD}$, pero que estos no reconocen como tales, sino que consideran como actividades rutinarias de clase. Se puede hipotetizar que esto ocurre a raíz de las teorías implícitas que los docentes tienen sobre este término en particular. Así, Pozo (1996) sostiene que la visión que un docente tiene sobre el aprendizaje a partir de las BPD puede fluctuar entre lo directivo, lo interpretativo o lo constructivista. La visión directa de las BPD tiene que ver con los resultados: en ese sentido, estos docentes ven a la BPD como un resultado en sí mismo a conseguir. De otro lado, los docentes con una visión interpretativa de las BPD conciben a estas como procesos, es decir, como una serie de acciones a realizar, pero comparten con los de la visión anterior el hecho de que esperan que, luego de este proceso, se obtengan una serie de resultados previamente considerados. Finalmente, la visión constructivista concibe a las BPD como una serie de pautas generales de acción que, en determinados contextos, permiten la consecución de una serie de resultados positivos; sin embargo, estos resultados concretos no son considerados como el objetivo central de las BPD, sino más bien el proceso de construcción y desarrollo general de los estudiantes, que requiere de la puesta en marcha, evaluación y reevaluación constante de las BPD. Dentro de este marco general, por tanto, es posible hipotetizar que los docentes entrevistados parecen reconocer el proceso de las BPD en sí, pero que esta no es una visión imperante en ellos.

Por otro lado, los docentes reconocen que las TIC son un elemento importante para el desarrollo de la educación; sin embargo, el contexto al que pertenecen no favorece el uso adecuado de estas en el salón de clase. De esta manera, es necesario que los estudiantes acudan a un salón aparte para hacer uso de tablets, computadoras, y otros elementos tecnológicos. Esto, según señalan los docentes, mengua el impacto de las TIC en el aprendizaje de los nińos, pues, debido a estas condiciones, separan en 2 días el esquema de clase, enfocándose en la explicación teórica el primer día y en la práctica con TIC el segundo día:

P: «Porque todo está inmerso en el mundo digital: no hay nada que no te funcione en la casa y en el entorno y en tus servicios y tus necesidades que 
ya se están convirtiendo en básicas, ¿no? Tú vas al banco y si no sabes digital no puedes sacar tu dinero, ¿no? Si tienes un celular y no sabes digital para contestar, para mandar mensaje, para utilizar uno de los aplicativos ahora de redes sociales. De una u otra manera, la sociedad te está exigiendo que entres a este mundo porque si no vas a estar, entre comillas, como informada y hasta inclusive desfasada» (participante 1)

P: «Son buenas herramientas, porque también pienso que a veces es mejor: los niños pareciera que sus celulares que tiene internet solo lo utilizan de manera social, ¿no? Pero, sin embargo, cuando uno lo usa en el aula aprende también a que no solamente es para cuestiones sociales, sino que también puede utilizarlo para investigar, para conocer, indagar, ¿no? Por ese lado me parece bueno» (participante 2).

E: «¿Identifica algún obstáculo en el uso de tecnologías en la clase?»

P: «[...] Sí estaría de acuerdo de repente en capacitación a los docentes en cuanto a la manipulación [de las tablets], porque de repente no todos saben» (participante 3)

Esta realidad puede ser contraproducente para los docentes, lo que exige pensar en estrategias diferentes para implementar el uso de estas herramientas. Existen experiencias previas, dentro del salón de clase, que pueden servir como ejemplo (Ortega, Lozano, \& Tristancho, 2015).

En relación con el aplicativo móvil Oráculo Matemágico, los docentes señalan que este motiva a los estudiantes a realizar más ejercicios matemáticos y fomenta la competitividad de los estudiantes debido al diseńo de la aplicación. Esto se debe a que Oráculo Matemágico ha sido diseñado en base a recompensas (útiles para el juego integrado a la aplicación) que son otorgadas al estudiante luego de que este resuelve algunos ejercicios matemáticos. Así, cumple con características reportadas por Young, Slota, Cutter, Jalette, Mullin, Lai, Simeoni, Tran y Yukhymenko (2012), quienes indican que los videojuegos tienen una influencia positiva en la motivación de los estudiantes, la cual se manifiesta en una mayor participación en clase. Además, los elementos de competitividad, reforzadores y recompensas, son importantes para mantener interesados a los estudiantes en las actividades gamificadas que están realizando (Huang y Soman, 2013). Los docentes reportan dos características particulares (obtención de monedas y obtención de Cartas Mágicas) del aplicativo móvil que van en la misma línea de lo antes mencionado:

P: «uy yo les digo bueno chicos ya saben que mañana nos toca ir al centro de recursos tecnológicos, weeee gritan o sino les digo mañana voy a venir a trabajar con ustedes con la tablet, yeeee responden (risas). Como te digo, es 
un espacio para que ellos puedan demostrarse qué tanto pueden hacer solos, es un espacio de oxigenación, de calma, de respeto a sus procesos. Donde la secuencia que haga o propongo y que lo lleva a lograr su objetivo, lo hace suyo, siente una satisfacción que lo hizo y al hacerlo en digital lo va a poder hacer en hoja, en la pizarra, en el mundo, ¿¿no? entonces es un momento muy bonito» (participante 2).

Asimismo, los docentes buscan diferentes maneras de utilizar este aplicativo con sus estudiantes, dejando que los estudiantes se equivoquen y que ellos mismos aprendan de sus errores.

P: «A veces yo les digo: llévate la respuesta y en tu casa vuelve a hacerlo, date cuenta dónde fallaste, pero hay pocos que lo hacen: señorita, sí me equivoqué en esto. Pero acá en el juego me he dado cuenta que ellos, para entrenar, hacen una parte que tú le pides que entrene como la intuición mágica y se han equivocado en algunas cosas, pero se les pide que lo vuelvan a hacer, ¿no? y en la segunda, en la tercera ya se dan cuenta y siguen haciendo lo mismo para ir acumulando sus monedas, entonces aprenden de sus errores y no se quedan con sus fallas como en el caso del salón: ah ya la señorita me ha dicho que lo revise, pero no lo hago. Acá por el afán de ganar, lo vuelven a hacer y lo vuelven a hacer, ¿no?» (participante 1)

En esta última cita, se puede evidenciar que los docentes han identificado el carácter motivacional del aplicativo. Los estudiantes se pueden equivocar; pero, debido a las características lúdicas del juego, quieren seguir intentándolo hasta lograr conseguir el objetivo. Asimismo, los docentes señalan diferencias importantes entre una clase tradicional y una clase con el aplicativo móvil Oráculo Matemágico:

P: «¿Entonces sería algo diferente si se tratara de ejercicios de matemática de hojas?

Obviamente porque eso es tedioso, es cansado para ellos, o sea para los chiquitos es realmente tedioso y cansado darles una ficha y ya desarrollen los ejercicios, o sea eso no les llama la atención, lo hacen de manera desmotivada y sí lo hacen, lo hacen por obligación porque tiene que ganar una nota. Pero en este caso, en los ejercicios que hay en el Oráculo Matemágico no, porque hay competencia entre ellos, ellos solos están: mire profesora ya tengo tantos puntos, están felices, el otro uy estoy menos» (participante 4).

Aquí puede señalarse que existe una experiencia gamificada de la enseñanza de matemáticas que no se había previsto, pero que es reportada como parte de las BPD por parte de los docentes. La gamificación (gamification) es la contextualización de una actividad no lúdica con elementos de un videojuego (Huang y Soman, 2013). Esto le adhiere un componente motivacional 
al aprendizaje de los estudiantes que tal vez no tendría con otras herramientas. Así, se puede identificar dos cosas: i) los reforzadores del videojuego influyen en la motivación de los estudiantes; ii) los docentes perciben este proceso como un elemento positivo que puede ser incluido en una BPD.

Los docentes refieren que los estudiantes se encuentran motivados para resolver una mayor cantidad de ejercicios matemáticos y, debido a esto, reportan notar mejoras en el cálculo mental, la comprensión de problemas matemáticos verbales, y que buscan más asesoramiento por parte del docente o sus compańeros, actitud que no había sido observada anteriormente:

P: «Bueno en el caso de ellos, lo que he observado es que el cálculo mental mejoró, en la segunda clase menos hoja de papel. Eso es lo primero y lo otro es que había una comunicación en lenguaje más matemático: ya no era «estito» sino decía "acá tenemos que adicionar», «buscar la relación por qué te ha ganado». Entonces el lenguaje se ha hecho más matemático y otro es la tolerancia a la frustración: «ya pues te ganó ahora me toca a mí». El otro es el diálogo entre pares, el que uno enseña al otro, la aceptación de que otro te corrija, que también es algo cultural, no nos gusta que nos digan «mira te equivocaste», eso también y a pesar que es un grupo medio movidito, son chicos bien 'hablantines', los míos no hablan mucho pero sí me gustó cuando uno le dijo «mira, acá te has equivocado», «no, pero está bien», le dijo, «pero cómo va a estar bien si estás viendo que este es menor y tú le has restado», «ah verdad, ¿¿no?». Entonces eso, la actitud al compañero, el respeto al que gana» (participante 2)

E: «QQué otro beneficio a nivel académico ha notado en los chicos? ¿Alguien ha mejorado su desempeño o cree que aún no hay un registro concreto?»

$\mathrm{P}:$ «De resultado, no, pero sí me doy cuenta de la rapidez mental. O sea, acá no te puedes demorar para dar la respuesta, acá no coges un papel y en el papel lo haces, porque también la matemática la tienes que hacer mentalmente, sobretodo el redondeo: el redondeo no es para que saques exactamente lo que te salió, sino para que intuitivamente y con las reglas del redondeo te aproximes. Entonces la agilidad mental también es algo bueno porque es rápido, tienes que avanzar, no te puedes quedar en ese problemita y después te das cuenta si lo hiciste bien, si lo hiciste mal» (participante 4).

De igual manera, algunos docentes señalan que no solo utilizan Oráculo Matemágico como motivación, sino también como herramienta a partir de los temas programados. Esto debido a que Oráculo Matemágico tiene la opción de elegir el tipo de ejercicios en su apartado de Entrenamiento:

P: «No habíamos visto, como lo tiene el Oráculo, un ángulo recto partido a la mitad, y el problema te daba acá 45 grados y preguntaba cuánto le falta a 
la otra parte. Entonces yo dudaba si empezar presentándoles el problema o no, pero lo hicimos y ellos iban tanteando. Algunos lo hicieron bien y otros me preguntaban 'pero señorita, ¿cómo vamos a ver la otra parte?'. Primero les pregunté ‘qué saben?, ¿cómo lo pueden resolver?’ Así que copiamos el problema en papelote y lo resolvimos juntos y luego volvimos al Oráculo y 'ahora sí, hazlo' (participante 1).

E: ««¿Cómo es un apoyo para usted [Oráculo Matemágico]? ¿En qué le facilita a usted como docente?»

$\mathrm{P}$ : «Por ejemplo, ahora en el trabajo de descomposición que he hecho, me facilita en el sentido de que me puedo guiar de ahí algunos ejercicios a desarrollar, ¿̨no? Y de cierta manera saber qué es lo que voy a preparar, qué material voy a preparar para la siguiente clase, entonces me guio un poco de ahí» (participante 3).

En tal sentido, se puede concluir que, si bien los docentes no identifican qué es una BPD a nivel práctico, sí utilizan actividades y/o acciones concretas que podrían considerarse como tales. Debido a ello, si bien han utilizado el aplicativo móvil (Oráculo Matemágico) como motivador y, en algunos casos, como herramienta, se les dificulta acoplar efectivamente el uso de esta TIC en el salón. Sumado a esto, el contexto en el cual están inmersos los docentes y estudiantes hace aún más difícil implementar el uso de TIC en las BPD, pues las limitaciones de hardware y de software influyen negativamente.

Asimismo, parece que los docentes conciben una relación entre las BPD y las estrategias de aprendizaje. Esto puede ser positivo si se quiere brindar un marco conceptual más amplio para el estudio de las BPD, pues permitiría alejarse del componente empresarial con el que se originó, otorgándole una base pedagógica que puede ser más útil si se quiere trabajar en un contexto educativo. Esta visión de las BPD debería ser estudiada en el futuro.

Por otro lado, los docentes reconocen la importancia de motivar a sus estudiantes al momento de enseñar matemáticas. En ese sentido, señalan la utilidad de diversas tecnologías (incluyendo el aplicativo Oráculo Matemágico) como un factor positivo. Asimismo, reconocen la funcionalidad de una herramienta gamificada para motivar a sus estudiantes. En ese sentido, se puede considerar que el uso de nuevas tecnologías puede ser un factor importante en las BPD para la enseñanza de matemáticas.

Finalmente, Oráculo Matemágico ha sido descrito como una herramienta útil y positivo. Ante esto, se puede concluir que, teniendo una base teórica adecuada con relación a BPD, complementandolo con un adecuado conocimiento sobre el uso de estrategias de enseñanza, y realizando una buena planificación de la misma e interviniendo eficazmente, Oráculo Matemágico puede ser utilizado como una herramienta motivadora y de uso práctico en el salón de clases. 


\section{Conclusiones}

Las buenas prácticas docentes son experiencias positivas que los docentes experimentan en su práctica pedagógica. Debido a su carácter innovador, el uso de TIC es comúnmente asociado a estas, donde se implementan con éxito en distintos contextos.

Este estudio permitió conocer la percepción de los docentes sobre las buenas prácticas con el uso de un aplicativo móvil para la enseñanza de matemáticas. El aplicativo móvil fue probado por los docentes, quienes reconocieron las virtudes y posibilidades de este tipo de materiales en el contexto educativo, particularmente para la enseńanza de matemáticas. Es importante el uso de TIC para la enseñanza, pues esto permite que los estudiantes desarrollen habilidades del siglo 21, necesarias para tener profesionales competentes que puedan enfrentarse a las necesidades del nuevo siglo (Ananiadou \& Claro, 2009; Voogt y Pareja, 2010).

Motivar y facilitar el aprendizaje de los estudiantes es una de las tareas más importantes de los docentes. Se espera que, con este tipo de iniciativas y aplicativos, los docentes puedan explorar y explotar más prácticas pedagógicas innovadoras que beneficien a sus estudiantes.

\section{REFERENCIAS BIBLIOGRÁFICAS}

Abdoulaya, A. (2003). Conceptualisation et dissemination des «bonnes pratiques» en éducation: essai d'une approche internationale à partir d'enseignements tirés d'un projet. Développement curriculaire et 'Bonne Pratique en education'. Ginebra: International Bureau of Education, 1-11.

Ananiadou, K. y M. Claro (2009). 21st Century Skills and Competences for New MillenniumLearnersin OECD Countries. OECDEducation WorkingPapers, No. 41, OECD Publishing. https://doi.org/10.1787/218525261154

Arnaiz, P. (2011). Luchando contra la exclusión: buenas prácticas y éxito escolar. Innovación Educativa, (21), 23-35.

Chickering, A. W. y Gamson, Z. (1987). Seven Principles for Good Practise in Undergraduate Education. American Association for Higher Education Bulletin (march): Washington, DC.

Colás, P. y Casanova, J. (2010). Variables docentes y de centro que generan buenas prácticas con TIC. Teoría de la Educación. Educación y Cultura en la Sociedad de la Información, 11(1), 121-147.

De Pablos, J. y González, T. (2007). Políticas educativas e innovación educativa apoyada en TIC; sus desarrollos en el ámbito autonómico. Comunicación 
Ricardo Javier Navarro Fernández et al.

publicada en las Actas de las II Jornadas Internacionales sobre politicas educativas para la sociedad del conocimiento. Granada.

De Pablos, J. y Jiménez, R. (2007). Buenas prácticas con TIC apoyadas en las Políticas Educativas: claves conceptuales y derivaciones para la formación en competencias ECTS. Revista Latinoamericana de Tecnología Educativa, 6(2), 15-28. http://campusvirtual.unex.es/cala/editio/

Epper, R. y Bates, A.W. (2004). Enseñar al profesorado cómo utilizar la tecnología. Buenas prácticas de instituciones líderes. Colección Educación y Sociedad Red. Barcelona: UOC.

Escudero, J. (2009). Buenas prácticas y programas extraordinarios de atención al alumnado en riesgo de exclusión educativa. Profesorado. Revista de Curriculum y Formación de Profesorado, 13(3), 107-141.

Fojtik, R. (2014). Mobile Technologies Education. Procedia-Social and Behavioral Sciences, 143, 342-346. https://doi.org/10.1016/j.sbspro.2014.07.417

Graells, P. M. (2013). Impacto de las TIC en la educación: funciones y limitaciones. 3 c TIC: cuadernos de desarrollo aplicados a las TIC, 2(1), 2.

Huang, W. H. Y. y Soman, D. (2013). Gamification of education. Research Report Series: Behavioural Economics in Action. Toronto, ON: Rotman School of Management, University of Toronto.

Keengwe, J. y Bhargava, M. (2014). Mobile learning and integration of mobile technologies in education. Education and Information Technologies, 19(4), 737-746. https://doi.org/10.1007/s10639-013-9250-3

Lourenco, O. L. O. (2015). Piaget's Legacy to Human Development. World Journal of Behavioral Science, 1, 53-65.

Mikre, F. (2011). The roles of information communication technologies in education: Review article with emphasis to the computer and internet. Ethiopian Journal of Education and Sciences, 6(2), 109-126.

Monereo, C. (2000). Estrategias de enseñanza y aprendizaje. Formación del profesorado y aplicación en la escuela. Barcelona: Graó.

Murillo, F. J., Martínez, C. A. \& Hernández, R. (2011). Decálogo para una enseñanza eficaz. REICE. Revista Iberoamericana sobre Calidad, Eficacia y Cambio en Educación, 9(1).

Noguera, I. (2015). How Millennials are changing the way we learn: the state of the art of ICT integration in education. RIED: Revista Iberoamericana de Educación a Distancia, 18(1), 45-65.

Ortega, M. V., Lozano, J. J. M. y Tristancho, S. L. Z. (2015). APPS en el rendimiento académico y autoconcepto de estudiantes de ingeniería/Apps in the academic achievement and engineering student self. Revista Logos Ciencia \& Tecnología, 6(2), 198-208. 
Piaget J. (1970). Science of education and the psychology of the child. Londres: Kegan Paul Trench Trubner.

Piaget, J. (1973). To understand is to invent: The future of education. Nueva York: Grossman Publishers.

Pozo, J. I. (1996). Estrategias de aprendizaje (Vol. 3552). En C. Coll (comp.), Desarrollo psicológico y educación II. Psicología de la educación. Madrid: Alianza.

Prieto, V., Quiñones, I., Ramírez, G., Fuentes, Z., Labrada, T., Pérez, H. O. y Montero, M. (2011). Impacto de las tecnologías de la información y las comunicaciones en la educación y nuevos paradigmas del enfoque educativo. Educación Médica Superior, 25(1), 95-102.

Vidal Ledo, M. y Morales, I. (2009). Buenas prácticas docentes. Educación Médica Superior, 23(1).

Voogt, J. (2003). Consequences of ICT for aims, contents, processes, and environments of learning. En J. van den Akker, W. Kuiper y U. Hameyer (eds.), Curriculum landscapes and trends (pp 217-236). Dordrecht: Kluwer Academic Publishers.

Voogt, J. y Pareja, N. (2010). 21st Century Skills. Discussion Paper. Enschede (The Netherlands): University of Twente.

Young, M. F., Slota, S., Cutter, A. B., Jalette, G., Mullin, G., Lai, B., Simeoni, Z., Tran, M. y Yukhymenko, M. (2012). Our princess is in another castle: A review of trends in serious gaming for education. Review of Educational Research, 82(1), 61-89. https://doi.org/10.3102/0034654312436980 